\title{
Are affluent, well-educated, career-orientated women knowledgeable users of the oral contraceptive pill?
}

\author{
Megan van der Westhuizen, David Hall
}

\section{Abstract}

Objective Studies have shown poor knowledge of oral contraceptives among women attending government health clinics and women in rural areas. Little is known about the level of contraceptive knowledge in educated, affluent, career-orientated women, although it could be expected that access to information would be greater. The study objective was to describe the profile, knowledge and understanding of oral contraceptive users in a private general practice in Johannesburg, South Africa.

Methods Over a period of 3 months, all women attending a private general practice who were using an oral contraceptive were asked to complete an anonymous questionnaire. Informed written consent was obtained in all cases.

Results Fifty-one women participated in the study. Most women were nulliparous $(71 \%)$, held a tertiary educational qualification $(80 \%)$, were employed $(84 \%)$ and were not concerned about the cost of their pill $(65 \%)$. Most respondents $(86 \%)$ obtained their information from a doctor. However, only $12 \%$ of women were aware of the danger of extending the active pill-free interval. Less than half $(49 \%)$ were aware that their pill was less effective if taken more than 12 hours late and only $31 \%$ of women knew that their pill was effective again after taking seven active tablets.

Conclusions Educated, affluent women attending a private general practice lacked basic knowledge of the oral contraceptive pill. Consultations by practitioners need to be improved.

J Fam Plann Reprod Health Care 2005; 31(4): 307-309 (Accepted 11 August 2005)

\section{Key message points}

- Most respondents (86\%) obtained their information from a doctor.

- Only $12 \%$ of women were aware of the danger of extending the active pill-free interval.

- Only $31 \%$ of women knew that after skipping a pill, efficacy was regained after taking seven consecutive active tablets.

- Well-educated women are not necessarily knowledgeable on effective pill use.
Department of Obstetrics and Gynaecology, Tygerberg Hospital and Stellenbosch University, Tygerberg, South Africa

Megan van der Westhuizen, MB BCh, General Practitioner and Postgraduate Student

David Hall, FCOG(SA), MD, Associate Professor and Principal Specialist

Correspondence to: Professor David R Hall, Department of Obstetrics and Gynaecology, Stellenbosch University and Tygerberg Hospital, PO Box 19063, Tygerberg 7505, South Africa. Tel: +27 21938 9059. Fax: +27 219322455

E-mail: drh@sun.ac.za

\section{Background}

Numerous studies have demonstrated that poor knowledge of the oral contraceptive pill (OCP) is common in women taking the pill and is associated with unwanted pregnancy. ${ }^{1-7}$ These studies investigated low-income, African-American adolescents, ${ }^{1}$ women in rural Bangladesh, ${ }^{2}$ university-aged females attending government family planning clinics, ${ }^{3}$ and women attending National Health Service facilities in the UK. ${ }^{4-6}$

While it could be expected that affluent, well-educated, career-orientated women might represent the most knowledgeable group of OCP users, the evidence is scarce. On the contrary, the situation might be that the supposed knowledge of such women is taken for granted and that they are actually not well informed.

The principal author is a general practitioner and postgraduate student who runs a private practice in an affluent suburb in Johannesburg, South Africa. The practice list contains approximately 4000 names comprising men, women and children. Most of the women attending this practice are well educated and have a career. Reliable family planning and effective contraceptive are therefore essential. When prescribing an OCP for the first time, a 30minute consultation is scheduled. This allows time to educate the user regarding the product. During follow-up visits women are invited to ask questions or discuss concerns, but no structured information is given. The purpose of this study was to describe the profile, knowledge and understanding of oral contraceptive users in the practice, as well as their understanding and knowledge of the OCP.

\section{Methods}

Over a period of 3 months (August-October 2004), all women visiting the practice who were using an oral contraceptive were asked to complete an anonymous structured questionnaire. The development of the questionnaire was guided by the aim of the study and previous instruments. It solicited information regarding the profile of the user and tested her knowledge regarding use of the contraceptive agent. Informed written consent was obtained and all women approached agreed to participate in the study.

Table 1 Characteristics of women included in the study

\begin{tabular}{lrr}
\hline Characteristic & $n$ & $\%$ \\
\hline Highest educational qualification & & \\
High school certificate & 10 & 20 \\
College diploma & 15 & 29 \\
University degree & 7 & 14 \\
Postgraduate degree & 19 & 37 \\
Tertiary qualification & 41 & 80 \\
Occupation & & \\
Working but not studying & 28 & 55 \\
Working and studying & 15 & 30 \\
Studying but not working & 5 & 10 \\
Neither working nor studying & 2 & 4 \\
Total working & 43 & 85 \\
Children (n) & & \\
0 & 36 & 70 \\
1 & 6 & 12 \\
2 & 7 & 14 \\
3 & 2 & 4 \\
\hline
\end{tabular}




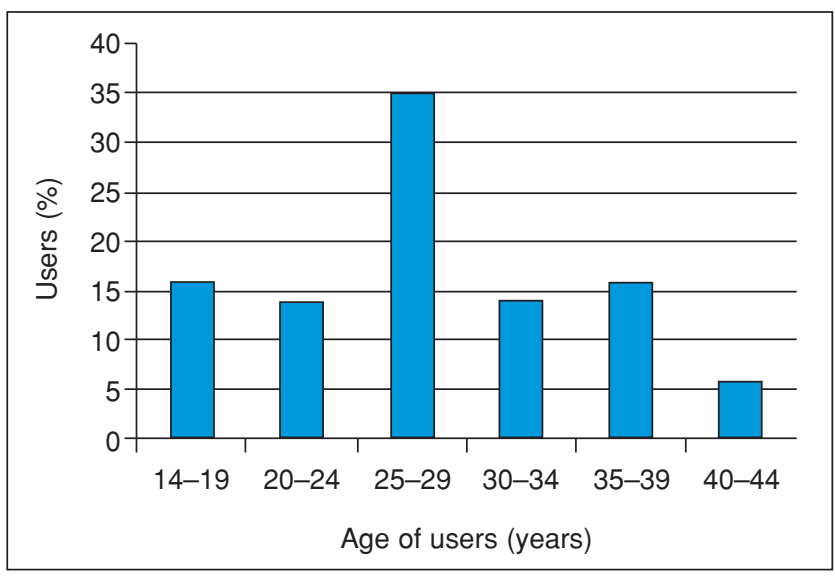

Figure 1 Age distribution of oral contraceptive users in the study

\section{Results}

All the women $(n=51)$ who were approached to participate completed the questionnaire (this high participation rate probably reflects the nature of the doctor-patient relationship in private practice). The profile of the contraceptive users is described below. The age of the contraceptive users ranged from 14 to 44 years with a median age of 27 years. Their age distribution is shown in Figure 1. The characteristics of the study participants are shown in Table 1.

Most of the women in the study $(n=48 ; 94 \%)$ were established OCP users. All women were asked whether the financial cost of their pill was a determining factor for them. Only $12(24 \%)$ women were concerned about the cost factor. Thirty-three $(65 \%)$ women were not concerned about the costs, while six (12\%) were undecided.

The replies to questions that tested knowledge related to the use of the OCP revealed the following. Only 25 (49\%) of women answered correctly that extra precautions needed to be taken for 7 days when the pill was taken more than 12 hours late. Eighteen (35\%) women placed this cut-off at 24 hours and eight (16\%) were uncertain. Women were asked to indicate which were the most important pills not to skip in terms of efficacy. Six (12\%) women answered correctly that this would be the first and/or last pill in the packet. Twenty-nine (57\%) women believed that all pills were equally important while seven $(14 \%)$ indicated that it would be most important not to skip a pill in the middle of the pack. Nine (18\%) women were uncertain of the answer. Knowledge of factors that may compromise the efficacy of the OCP is shown in Figure 2.

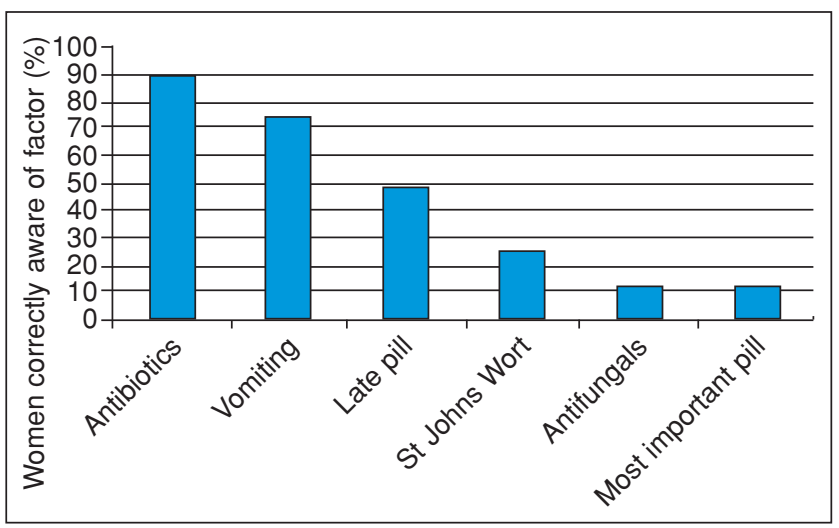

Figure 2 Knowledge of factors that may compromise the efficacy of the contraceptive pill
When asked when their pill would be effective again after skipping a tablet, or after compromise by vomiting or antibiotics, $16(31 \%)$ women answered correctly that the pill would regain efficacy after seven active tablets. Sixteen $(31 \%)$ women replied that the pill would be effective again after taking 14 active tablets while $11(22 \%)$ answered that the pill would only be effective after taking a whole pack. Eight $(16 \%)$ women were unsure. In answer to the question as to whether they were using a low- $(\leq 35 \mu \mathrm{g}$ ethinylestradiol) or high-dose (50 $\mu \mathrm{g}$ ethinylestradiol) pill, 20/22 women correctly identified their pill as a low-dose product. All eight women who thought they were taking a high-dose OCP were correct. However, 21 (41\%) women were uncertain.

The sources of information for the participants were tested. Only seven (14\%) women did not regard their doctor as the most important source of information. In fact, $38(75 \%)$ women obtained their information solely from their doctor.

When asked why they had chosen their particular formulation of contraceptive pill, 40 (78\%) women indicated that they had been advised by their doctor, whereas only four $(8 \%)$ had been advised by a family planning clinic. Six (12\%) women had chosen their pill in order to treat acne and one respondent was uncertain. Finally, the questionnaire investigated whether the user had ever changed her OCP and the reason for the change. Most $(61 \%)$ women indicated they had changed their pill and six (12\%) women had changed their pill more than once.

\section{Discussion}

The results showed that the typical OCP user in this practice at the time of the study was a nulliparous woman in her twenties with a tertiary educational qualification who was employed and for whom the cost of the contraceptive was not a factor. Such career-orientated women require reliable contraception but the reliability of the OCP is also user dependent. This means that women must possess basic core knowledge related to everyday use of the pill. It seems reasonable to expect that well-educated and well-motivated women would be well informed but such knowledge was sadly lacking in this study. In contrast, Smith and Whitfield ${ }^{6}$ found that user knowledge of the OCP was higher among women attending an urban medical practice. A recent study amongst adolescents at an urban public hospital in El Salvador ${ }^{8}$ found that education and literacy predicted contraceptive knowledge; however, a questionnaire distributed to female university students in Canada revealed that they might not be equipped with adequate knowledge to practise efficacious pill use. ${ }^{3} \mathrm{~A}$ limitation of our study is that it was not a large one. This precluded the comparison of various subgroups in the analysis.

How could the lack of knowledge demonstrated in this study be improved? Some $86 \%$ of women in the study obtained their information regarding oral contraceptives from their doctor, while $78 \%$ had indicated that they had chosen to take their particular pill on the advice of their doctor. It is surprising that this group of women with easy access to information would rely so heavily on their doctors. However, better transfer of information from the doctor or other medical personnel to the patients would probably have benefited this group of women. This could be effected by counselling and provision of information sheets and package inserts, if they are read. Despite the fact that package inserts provide information regarding the " 7 day rule', $69 \%$ of women in the study were unaware that their pill would be effective again after taking seven consecutive active tablets. 
Little et al. performed a randomised controlled trial amongst patients attending general practices in England. 4 They showed that knowledge of factors compromising efficacy of the pill was good, but that knowledge of what action to take subsequently was poor. They concluded that women attending checkups for repeat prescriptions of the pill should be provided with educational leaflets to improve their knowledge. When this was combined with asking relevant questions the most knowledge was gained, although asking questions was more time consuming. Gilliam et al. studied a different group of women. ${ }^{1}$ They recruited 43 African-American adolescents and young adults from a low socioeconomic group. After one session of standardised counselling the participants completed a series of questions regarding importance, confidence and knowledge of oral contraceptives. Their results showed that despite high motivation and confidence in their ability to take the pill, many women did not fully understand the information that they had received. The important point for caregivers here is that motivation and confidence to use the OCP do not imply that there is sufficient knowledge of factors causing pill failure, subsequent action and emergency contraception.

In the results reflecting knowledge of factors that may compromise the efficacy of the OCP only $12 \%$ of women indicated that antifungals interfere with efficacy of the OCP. This figure should be interpreted with caution, as the question did not specify griseofulvin, which is the only antifungal agent that has been proven to interfere with efficacy of the OCP. ${ }^{9}$

In conclusion, well-educated, affluent women attending a private general practice lacked basic knowledge of the OCP. Initial and repeat consultations by doctors need to be improved. In South Africa, the recent reinforcement of the regulation requiring a prescription for an oral contraceptive provides doctors with an opportunity every 6 months to further patient education regarding the pill.

Statements on funding and competing interests

Funding. None identified.

Competing interests. None identified.

References

Gilliam ML, Knight S, McCarthy M. Importance and knowledge of oral contraceptives in antepartum, low-income, African American adolescents. J Pediatr Adolesc Gynecol 2003; 16: 355-360.

2 Saha U, Khan M, Begum M, Bairagi R. Determinants of pill failure in rural Bangladesh. J Biosoc Sci 2004; 36: 39-50.

3 Fletcher PC, Bryden PJ, Bonin E. Preliminary examination of oral contraceptive use among university-aged females. Contraception 2001; 63: 229-233.

4 Little P, Glew C, Kelly J, Griffin S, Dickson N, Sadler C. Contraceptive knowledge: development of a valid measure and survey of pill users and general practitioners. Br J Fam Plann 1998; 24: $98-100$.

5 Little P, Griffin S, Kelly J, Dickson N, Sadler C. Effect of educational leaflets and questions on knowledge of contraception in women taking the combined contraceptive pill: randomised controlled trial. BMJ 1998; 316: 1948-1952.

6 Smith L, Whitfield M. Women's knowledge of taking oral contraceptive pills correctly and of emergency contraception: effect of providing information leaflets in general practice. $B r J$ Gen Pract 1995: 45: 409-414.

7 Beksinska ME, Rees VH, Nkonyane T, McIntyre JA. Compliance and use behaviour, an issue in injectable as well as oral contraceptive use? A study of injectable and oral contraceptive use in Johannesburg. $\mathrm{Br}$ J Fam Plann 1998: 24: 21-23.

8 Newmann SJ, Goldberg AB, Aviles R, Molina de Perez O, FosterRosales AF. Predictors of contraception knowledge and use among postpartum adolescents in El Salvador. Am J Obstet Gynecol 2005; 192: 1391-1394.

9 Faculty of Family Planning and Reproductive Health Care Clinical Effectiveness Unit. FFPRHC Guidance (April 2005). Drug interactions with hormonal contraception. J Fam Plann Reprod Health Care 2005; 31: 139-151.

\section{But is she positive?}

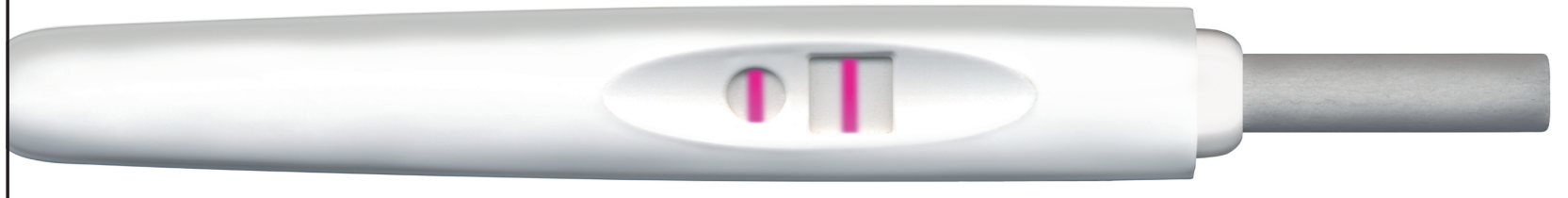

Your next patient is pregnant but doesn't want to be.

Where next? As a registered charity since 1968, bpas has offered affordable abortion care for women. We provide almost 50,000 abortions a year (including service agreements) and can offer all the professional help your patient needs.

bpas has a nationwide network of clinics and consultation centres. There are no long waits for appointments. We can offer a choice of times, clinics and procedures. All it takes to arrange an appointment is one call to the bpas Actionline on 08457304030 .

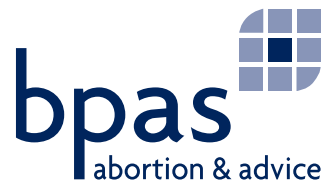

ACTIONLINE 08457304030

bpas positively the best service 